\title{
Adaline for Online Symmetrical Components and Phase Angles Identification in Transmission Lines
}

\author{
Fatima Louisa Yousfi, Djaffar Ould Abdeslam, Member, IEEE, Tahar Bouthiba, Ngac-Ky Nguyen, and \\ Jean Mercklé
}

\begin{abstract}
This paper presents a new method for online symmetrical components and phase angles extraction from High Voltage (HV) transmission line faults. This method is based on the Adaline neural networks and the instantaneous power theory (IPT), also known as the $p-q$ method. A new current decomposition is proposed in order to derive the direct, inverse and homopolar current components. The average and oscillating terms of powers in the $\alpha \beta$ frame are separated by using four Adaline neural networks. The Adalines use a cosine and sine as inputs in order to learn the linear combination of the powers. The resulting symmetrical components are used by three other Adalines for phase angles estimation between direct and inverse current components. These phase angles permit to classify the fault types. The neural networks use an online learning process based Widrow-Hoff algorithm and can adapt their weight parameters to the power supply evolution. Simulation results show the performance and the robustness of this method and provide a perspective for protection relay improvement.
\end{abstract}

Index Terms-Transmission Line Protection, Artificial Neural Networks, Adaline, Instantaneous Power Theory, Symmetrical Components.

\section{INTRODUCTION}

$\mathbf{I}^{\mathrm{N}}$ $\mathrm{N}$ order to generate electrical power and transmit it over long distances to customers, a large amount of capital investment is made to make the power system operate at peak efficiency. Electrical transmission lines, which have an important role in the electrical supply, are subject to various disturbances. The most frequent are climate-induced, such as short circuits caused by lightning, phase failure due to falling trees, etc.

The protective relay is used to safeguard the HV transmission lines from these accidents [1]. This protective solution is composed of three principal modules. The first module, "Fault detection", gives a signal to subsequent modules that a fault has occurred. The second module, "Fault classification", estimates which phases are affected and outputs the fault type. The last module, "Fault location", determines whether the fault area has been affected by faults. The fault detector and classifier modules are important devices and constitute an essential part of the protection algorithm. Any error in the fault type detection and classification may lead to the misoperation

F.L. Yousfi, D. Ould Abdeslam, N.K. Nguyen and J. Mercklé are with MIPS laboratory, University of Haute Alsace, 68093 Mulhouse Cedex, France (e-mail: yflouisa@gmail.com; djaffar.ould-abdeslam@uha.fr; ngacky.nguyen@uha.fr; jean.merckle@uha.fr). F.L. Yousfi permanent affiliation is same as T. Bouthiba.

T. Bouthiba is with the Department of Electrical Engineering, University of Technology, BP 1505, 31000 El Menaouer, Oran, Algeria (e-mail: tbouthiba@yahoo.com).

Manuscript received $* * * * * *$. of the protection system. Thus, fast, selective, accurate and reliable protective relay improves the quality of the power system.

Conventional algorithms for fault protection are based on the decomposition of unbalanced three phase voltages or currents into symmetrical components. These components are calculated with a linear method of relatively short delay, such as discrete Fourier analysis [2]. Unfortunately, at the appearance of the faults there are transients in the measured signals and thus, the symmetrical components are determined with errors. To overcome most of the problems related to conventional algorithms, adaptive protection concepts were proposed to make relays adaptive to changing system conditions and improve their performances [3].

Over last decades, many researchers have studied the application of Artificial Intelligence to protection systems. As a result, an important number of fault protection based on these methods have been developed: Expert Systems [4], [5], Fuzzy Logic [6]-[8], Wavelet Transform [9], [10], Artificial Neural Networks (ANNs) [17]-[32], etc. Among these intelligent methods, neural networks are the most applied. This is due to their generalization capability, noise immunity and are expected to be more accurate for the system condition variations. Some of them are presented in the next section. In this paper, we propose an improved Adaline neural networks method for online transmission line protection. A new current signals decomposition method is developed. Based on the instantaneous power theory [14], [15], online symmetrical components and phase angles algorithms are developed. The IPT method also known as the $p-q$ theory uses the Clarke transformation, which consists of a real matrix that transforms three phase voltages and currents signals into the $\alpha \beta$ stationary reference frames. This method is valid not only in the steady state, but also in the transient state. It is also appropriate for distorted voltages under unbalanced and non stationary conditions.

This paper contains six sections. The state of the art of neural networks applications in power systems fault protection is discussed in section II. Section III details the symmetrical components extraction by using the IPT approach based on Adaline neural networks. A fault classification approach by using Adaline neural networks is presented in section IV. Simulation results and related discussions are given in the section V. Finally, section VI concludes the paper. 


\section{STATE OF ART OF TRANSMISSION LINES PROTECTION USING ARTIFICIAL NEURAL NETWORKS}

Artificial neural networks have been successfully used in several engineering applications. In recent years, this artificial intelligence technique is increasingly used in the electrical supply field [16]. The fault protection of the transmission lines is directly concerned by these developments. There are several studies that use ANNs for fault detection, classification and location. In this section we present the neural networks architectures most commonly applied: MultiLayer Perceptron (MLP), Radial Basis Function (RBF), Neuro-Fuzzy (NF) and Adalines.

The most popular ANN, the MultiLayer Perceptron, with the Back-Propagation algorithm, is used to classify different fault types in a transmission line [17]-[20]. Promising results are obtained due to the good generalization ability of the MLP and its ease of use. Its major drawback is the difficulty to determine the number of hidden layers and neurons. In [21], a comparison between the MLP and another ANN, called SelfOrganizing Map (SOM), is described. The authors conclude that the SOM doesn't need large training sets. It is more robust to parameter variations such as fault location, fault resistance, fault inception angle, source impedance and load angle changes.

A Radial Basis Function is a type of single hidden layer ANNs. The activation functions in the hidden and the output layer neurons are Gaussian and linear, respectively. The RBF has more advantages, in comparison to the MLP, in terms of classification capabilities. In [22], the number of nodes in the hidden layer of the RBF is reduced by using the Orthogonal Least Square (OLS) algorithm. The authors make a comparison with a MLP and simulation results show that the RBF approach gives better performances. In [23], the $\mathrm{RBF}$ is compared with 4 other neural networks: MLP, Feature Map, Counter Propagation and Learning Vector Quantization. The Authors conclude that the RBF is better in terms of computation, number of training data used, learning speed and misclassification rate. Another variant of RBF called Minimal Radial Basis Function Neural Network (MRBFNN) for distance protection is used in [24]. The number of hidden layer neurons is optimized by using a sequential learning algorithm based on the iterated Kalman Filter. The obtained neural approach reduces the number of training patterns and the training time.

The association of neural networks with fuzzy logic introduces a human operator knowledge and improves the resulting architecture. The authors in [25] classify the faults with a Neuro-Fuzzy approach using symmetrical components as inputs. An advanced Adaptive Resonance Theory (ART) based neural network algorithm combined with Fuzzy Logic has been introduced in [26]. The algorithm shows superior classification capabilities compared to basic version of the ART algorithm, MLP neural network algorithm, and impedance based distance relay algorithm. An Adaptive Network and Fuzzy Inference System (ANFIS) is proposed in [27]. The ANFIS can detect and classify faults including low and high impedance faults accurately within half a period. It is also used to detect the fault placement in a combined overhead transmission line with underground power cable [28]. A Wavelet Neuro-Fuzzy combined approach for digital relaying is presented in [29]. The authors use a Wavelet Transform with an ANFIS for fault classification and location. The algorithm is independent of the fault location. In [30], a comparison is made between the ANFIS, the MLP and the cubic polynomial interpolation for fault location estimation. The authors conclude that the ANFIS architecture produces the most accurate results.

The Adaline is another adaptive neural network that has been employed for various types of applications. The most interesting in power system fields are harmonics estimation and active power filtering. In [31], the Adaline is developed for an on line faults detection in the EHV transmission lines. The adaptive neural network is used in the current frame and tracks the fundamental and DC component currents. It is compared with the MLP in terms of fault time detection. In [32], the Adaline is developed as an adaptive predictor to detect permanent and transient faults for single pole autoreclosers. By using voltage signals, the algorithm is able to estimate the secondary arc extinction time in case of the transient fault.

This state of the art shows that neural networks have been applied to detection, classification and location in the area of the transmission lines protection. The choice of an adequate ANN type depends on the desired objectives. Much research uses an MLP for its popularity and its ease to build but the main drawback of this ANN is the high computational cost. By contrast, the Adaline has a simple structure (a single neurone) and his online training has a very low computational cost. It is able to detect quickly any changes on the input signals. These advantages justify the integration of the Adaline in our development. Our work is based on the use of the Adalines for the detection and the classification of the HV transmission line faults. We include four Adalines in the $p-q$ method to extract the symmetrical components. Three other Adalines use the identified symmetrical components for phase angles estimation in order to classify the faults.

\section{ADALINES FOR SYMMETRICAL COMPONENTS EXTRACTION}

\section{A. Direct current components computation}

"Direct, inverse and homopolar" terminology is used instead "positive, negative and zero" terminology.

The symmetrical component extraction strategy is based on the Instantaneous Powers Theory (IPT) [14]. According to this theory, the $p-q$ powers are calculated and their oscillating and average terms are instantaneously separated. The direct average terms of powers $\left(\bar{p}_{d}\right.$ and $\left.\bar{q}_{d}\right)$ are used to compute the three phase direct current components. The three phase inverse current components are also computed by using the inverse average terms of powers $\left(\bar{p}_{i}\right.$ and $\left.\bar{q}_{i}\right)$. Figure 1 shows the complete strategy of direct and inverse symmetrical components extraction.

We consider the following three phase current expression: 


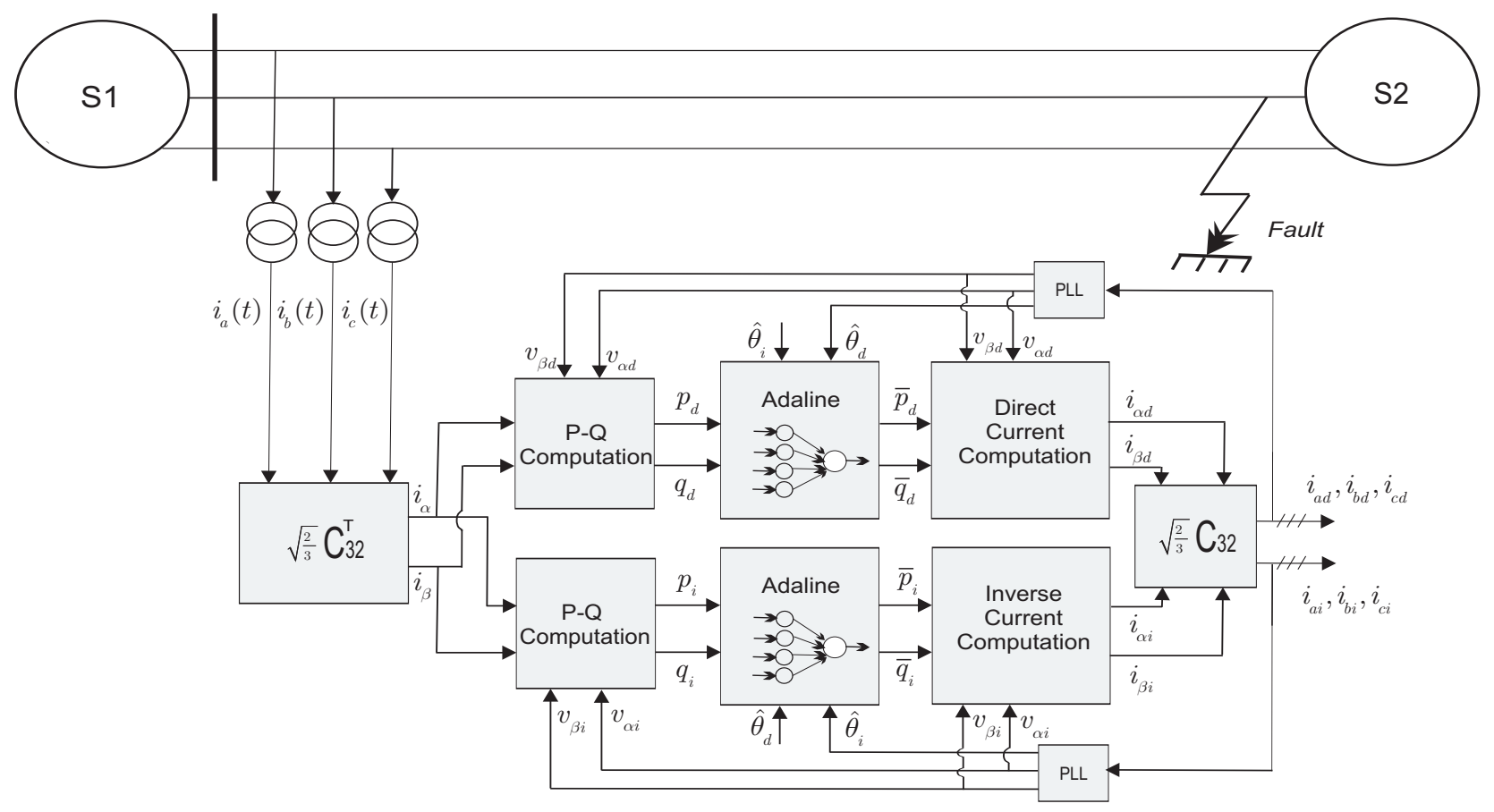

Fig. 1. System under study with the block diagram of the direct and inverse current components extraction based Adalines.

$$
\begin{aligned}
\mathbf{i}_{a b c}=\sum_{n=1}^{N} & \left(\sqrt{2} I_{d n} \mathbf{C}_{\mathbf{3 2}} \mathbf{P}\left(n \theta_{d}\right)\left[\begin{array}{ll}
1 & 0
\end{array}\right]^{T}\right. \\
& +\sqrt{2} I_{i n} \mathbf{C}_{\mathbf{3 2}} \mathbf{P}\left(-n \theta_{i}\right)\left[\begin{array}{ll}
1 & 0
\end{array}\right]^{T} \\
& \left.+\sqrt{2} I_{0 n} \mathbf{C}_{\mathbf{3 1}} \cos \left(n \theta_{0}\right)\right)
\end{aligned}
$$

where $\mathbf{C}_{32}$ and $\mathbf{C}_{31}$ are the Clarke matrix, $\mathbf{P}(\theta)$ is the Park matrix and $n$ is the harmonic range. $\theta_{k}=\omega_{k} t+\delta_{k}$ is the current instantaneous phase with $k=d, i, 0, \omega_{k}=2 \pi f$ is the frequency and $\delta_{k}$ is the initial phase. The index $d, i, 0$ mean direct, inverse and homopolar sequences. $I_{d n}, I_{i n}$ and $I_{o n}$ are the direct, inverse and homopolar current amplitudes.

The three-phase voltage can be defined similarly by replacing $\theta_{k}$ with $\theta_{k}^{\prime}=\theta_{k}+\phi_{k}$ where $\phi_{k}$ is the phase shift between the current and the voltage.

In the IPT, the instantaneous powers are calculated in the $\alpha \beta$ frame by the expression :

$$
\left[\begin{array}{c}
p \\
q
\end{array}\right]=\left[\begin{array}{cc}
v_{\alpha} & v_{\beta} \\
v_{\beta} & -v_{\alpha}
\end{array}\right]\left[\begin{array}{c}
i_{\alpha} \\
i_{\beta}
\end{array}\right]
$$

with

$$
\begin{aligned}
{\left[\begin{array}{l}
i_{\alpha} \\
i_{\beta}
\end{array}\right] } & =\sqrt{\frac{2}{3}} \mathbf{C}_{\mathbf{3 2}}^{\mathbf{T}} \cdot \mathrm{i}_{a b c} \\
& =\sum_{n=1}^{N}\left(\sqrt{3} I_{d n} \mathbf{P}\left(n \theta_{d}\right)\left[\begin{array}{ll}
1 & 0
\end{array}\right]^{T}\right. \\
& \left.+\sqrt{3} I_{i n} \mathbf{P}\left(-n \theta_{i}\right)\left[\begin{array}{ll}
1 & 0
\end{array}\right]^{T}\right)
\end{aligned}
$$

The active power $p$ is constituted by the average and oscillating components:

$$
p=\bar{p}_{d}+\bar{p}_{i}+\widetilde{p}_{d}+\widetilde{p}_{i}+\widetilde{p}_{d i}+\widetilde{p}_{i d}
$$

where $\bar{p}_{d}$ the average active power resulting from the direct components of voltages and currents. $\bar{p}_{i}$ the average active power resulting from the inverse components of voltages and currents. $\widetilde{p}_{d}$ the oscillating active power resulting from the direct components of voltages and currents. $\widetilde{p}_{i}$ the oscillating active power resulting from the inverse components of voltages and currents. $\widetilde{p}_{d i}$ and $\widetilde{p}_{i d}$ the oscillating active powers resulting from the combined direct and inverse components of voltages and currents.

By using the voltages issued from the direct fundamental system and the average terms of the associated powers, the fundamental direct currents in the $\alpha \beta$ frame are deduced from expression (2) as:

$$
\left[\begin{array}{c}
i_{\alpha d} \\
i_{\beta d}
\end{array}\right]=\frac{1}{\left(v_{\alpha d}^{2}+v_{\beta d}^{2}\right)}\left[\begin{array}{cc}
v_{\alpha d} & v_{\beta d} \\
v_{\beta d} & -v_{\alpha d}
\end{array}\right]\left[\begin{array}{c}
\bar{p}_{d} \\
\bar{q}_{d}
\end{array}\right]
$$

To extract the fundamental positive sequence current with the IPT method shown in figure 1, the amplitude of the fundamental direct voltages $v_{\alpha d}$ and $v_{\beta d}$ can be chosen arbitrarily. It is assumed that these voltages with any amplitude are derived only from an auxiliary positive sequence voltage at the estimated instantaneous phase $\hat{\theta}_{d}$ detected by the Phase Locked Loop (PLL) circuit [15]. For simplicity, these voltage amplitudes are set to unity and their phases are equal to zero in the $\alpha \beta$ frame, and are given by:

$$
\left[\begin{array}{l}
v_{\alpha d} \\
v_{\beta d}
\end{array}\right]=\left[\begin{array}{c}
\cos \left(\hat{\theta}_{d}\right) \\
\sin \left(\hat{\theta}_{d}\right)
\end{array}\right]
$$

The direct average terms of powers $\bar{p}_{d}$ and $\bar{q}_{d}$ are estimated with Adaline neural networks detailed in the next section. Thus, the direct current components $i_{a d}, i_{b d}, i_{c d}$ are recovered by converting the continuous powers in the $\alpha \beta$ current space 
with equation (5) and by multiplying them with the inverse Clarke transformation:

$$
\left[\begin{array}{lll}
i_{a d} & i_{b d} & i_{c d}
\end{array}\right]^{T}=\sqrt{\frac{2}{3}} \mathbf{C}_{32}\left[\begin{array}{ll}
i_{\alpha d} & i_{\beta d}
\end{array}\right]^{T}
$$

\section{B. Adaline neural networks for powers calculation}

The currents $i_{\alpha}$ and $i_{\beta}$ are used with the fundamental direct voltages $v_{\alpha d}$ and $v_{\beta d}$ to compute the auxiliary powers $p_{d}$ and $q_{d}$. These powers are based on measured currents $\mathbf{i}_{a b c}$ and direct voltages $v_{\alpha d}$ and $v_{\beta d}$, they are given by:

$$
\left[\begin{array}{c}
p_{d} \\
q_{d}
\end{array}\right]=\left[\begin{array}{c}
v_{\alpha d} i_{\alpha}+v_{\beta d} i_{\beta} \\
v_{\beta d} i_{\alpha}-v_{\alpha d} i_{\beta}
\end{array}\right]
$$

The direct active power $p_{d}$ can be detailed as follows:

$$
\begin{aligned}
p_{d} & =v_{\alpha d} \cdot i_{\alpha}+v_{\beta d} . i_{\beta} \\
& =3 I_{d 1} \cos \left(\phi_{d 1}\right)+\sum_{n=2}^{N} 3 I_{d n} \cos \left((n-1) \hat{\theta}_{d}+\phi_{d n}\right) \\
& -\sum_{n=1}^{N} 3 I_{i n} \cos \left(\left(n \hat{\theta}_{i}+\hat{\theta}_{d}\right)+\phi_{i n}\right)
\end{aligned}
$$

By developing expression (9), we obtain the separated direct active power components:

$$
p_{d}=\bar{p}_{d}+\widetilde{p}_{d}+\widetilde{p}_{d i}
$$

with:

$$
\bar{p}_{d}=3 I_{d 1} \cos \left(\phi_{d 1}\right)
$$

$\widetilde{p}_{d}=\sum_{n=2}^{N}\left[\begin{array}{ll}\cos (n-1) \hat{\theta}_{d} & \sin (n-1) \hat{\theta}_{d}\end{array}\right]\left[\begin{array}{c}3 I_{d n} \cos \phi_{d n} \\ -3 I_{d n} \sin \phi_{d n}\end{array}\right]$

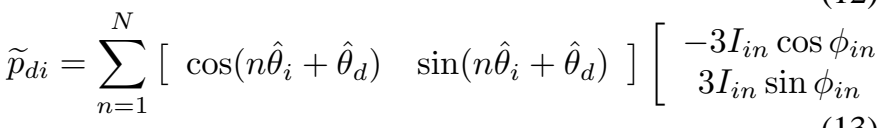

Expression (10) can be written with help of a vector product as a linear equation:

$$
y=\mathbf{W}^{T} \mathbf{X}
$$

with

$$
\mathbf{W}=\left[\begin{array}{c}
3 I_{d 1} \cos \phi_{d 1} \\
-3 I_{d 1} \sin \phi_{d 1} \\
-3 I_{i 1} \cos \phi_{i 1} \\
3 I_{i 1} \sin \phi_{i 1} \\
\vdots \\
3 I_{d n} \cos \phi_{d n} \\
-3 I_{d n} \sin \phi_{d n} \\
-3 I_{i n} \cos \phi_{i n} \\
3 I_{i n} \sin \phi_{i n}
\end{array}\right] ; \mathbf{X}=\left[\begin{array}{c}
1 \\
0 \\
\cos \left(\hat{\theta}_{i}+\hat{\theta}_{d}\right) \\
\sin \left(\hat{\theta}_{i}+\hat{\theta}_{d}\right) \\
\vdots \\
\cos (n-1) \hat{\theta}_{d} \\
\sin (n-1) \hat{\theta}_{d} \\
\cos \left(n \hat{\theta}_{i}+\hat{\theta}_{d}\right) \\
\sin \left(n \hat{\theta}_{i}+\hat{\theta}_{d}\right)
\end{array}\right]
$$

The Adaline neural approach presented in figure 2 learns vector expression (14). The Adaline is single neuron with multiple inputs, a single output and has a linear activation function. It was first proposed by Widrow and Hoff who developed the corresponding learning algorithm [33]. The input vector $\mathbf{X}$ is constituted of multiple sinusoidal signals of the direct active power $p_{d}$, the weight vector $\mathbf{W}$ is constituted of the amplitudes of $p_{d}$ and $y$ is the output of the Adaline. The Adaline is trained by supervised learning algorithm. During the training process, input patterns and corresponding desired responses are presented to it. The Adaline output is compared to a desired value which is $p_{d}$ obtained with the auxiliary voltages and measured currents $\mathbf{i}_{a b c}$. The error $\varepsilon=p_{d}-y$ is used to learn the Adaline weights by the Widrow-Hoff delta rule algorithm:

$$
\mathbf{W}(k+1)=\mathbf{W}(k)+\Delta \mathbf{W}(k)
$$

with:

$$
\Delta \mathbf{W}(k)=\frac{\alpha \varepsilon(k) \mathbf{X}(k)}{\lambda+\mathbf{X}^{T}(k) \mathbf{X}(k)}
$$

where $\alpha$ is the learning rate, $\mathbf{X}(k)$ is the input vector at instant $k$ and $\lambda$ is a constant chosen carefully to avoid of division by zero.

This algorithm minimizes the cost function over the training set, and the error is therefore reduced by equation (17) proportional to the learning rate $\alpha$. The choice of this learning rate $\alpha$ controls stability and the speed of convergence. The learning rate value is generally chosen between 0 and 1 . After training, the elements of the weight vector $\mathbf{W}$ represent power amplitudes. Finally, the average term of the instantaneous power $\bar{p}_{d}$ is obtained by using the first element of the weight vector $W_{1}$ that corresponds to the fundamental component $n=1$ [figure 2]. Another Adaline similar to the former is used to estimate the auxiliary reactive power $q_{d}$. Its output is compared with the desired value obtained from the expression (8) and after training, the average term $\bar{q}_{d}$ is obtained by using the first element of the weight vector.

In the same manner, the inverse current components are estimated by using the above developments and the fundamental inverse voltage defined by equation (18).

$$
\left[\begin{array}{l}
v_{\alpha i} \\
v_{\beta i}
\end{array}\right]=\left[\begin{array}{c}
\cos \left(\hat{\theta}_{i}\right) \\
-\sin \left(\hat{\theta}_{i}\right)
\end{array}\right]
$$

The fundamental direct and inverse current components $\mathbf{i}_{a b c d}$ and $\mathbf{i}_{a b c(i)}$ can be used to deduce the homopolar current components $\mathbf{i}_{a b c(0)}$.

\section{ADALINES FOR FAULT CLASSIFICATION BY USING SYMMETRICAL COMPONENTS}

The analysis of the fault current symmetrical components provides information on the nature of the fault. The presence of only the inverse component in the fault current indicates that a line to line fault has occurred. The presence of inverse and homopolar components indicates that a fault of single line to ground or double line to ground has occurred. Under normal or symmetrical fault conditions (three line to ground), the homopolar and inverse components in the line currents are nearly zero. The fault classification based on symmetrical components have been proposed in [7], [25]. 


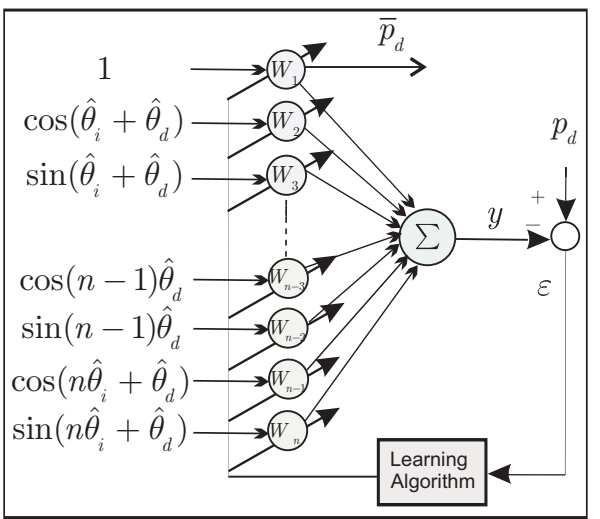

Fig. 2. Adaline for direct active power estimation.

In this section we present an Adaline neural network based approach to calculate the angular phases between direct and inverse current components. These angular phases serve to classify fault types in a HV transmission line.

\section{A. Adaline for phase angle estimation}

Consider the fundamental direct and inverse current components given by the following expressions :

$$
\begin{gathered}
i_{d}(t)=I_{d} \sin \left(\hat{\theta}_{d}+\phi_{d}\right) \\
i_{i}(t)=I_{i} \sin \left(\hat{\theta}_{i}+\phi_{i}\right)
\end{gathered}
$$

The product $i_{d}(t) \cdot i_{i}(t)$ of these signals is :

$$
\begin{aligned}
i_{d}(t) \cdot i_{i}(t)= & \frac{I_{d} \cdot I_{i}}{2}\left(\cos \left(\left(\hat{\theta}_{d}-\hat{\theta}_{i}\right)+\left(\phi_{d}-\phi_{i}\right)\right)\right. \\
& \left.-\cos \left(\left(\hat{\theta}_{d}+\hat{\theta}_{i}\right)+\left(\phi_{d}+\phi_{i}\right)\right)\right)
\end{aligned}
$$

The development of equation (21) gives a summation expression formed by sine and cosine terms as:

$$
\begin{aligned}
i_{d}(t) \cdot i_{i}(t) & =c_{1} \cos \left(\hat{\theta}_{d}-\hat{\theta}_{i}\right)-c_{2} \sin \left(\hat{\theta}_{d}-\hat{\theta}_{i}\right) \\
& -c_{3} \cos \left(\hat{\theta}_{d}+\hat{\theta}_{i}\right)+c_{4} \sin \left(\hat{\theta}_{d}+\hat{\theta}_{i}\right)
\end{aligned}
$$

with $c_{1}=\frac{I_{d} \cdot I_{i}}{2} \cos \left(\phi_{d}-\phi_{i}\right), c_{2}=\frac{I_{d} \cdot I_{i}}{2} \sin \left(\phi_{d}-\phi_{i}\right), c_{3}=$ $\frac{I_{d} \cdot I_{i}}{2} \cos \left(\phi_{d}+\phi_{i}\right)$ and $c_{4}=\frac{I_{d} \cdot I_{i}}{2} \sin \left(\phi_{d}+\phi_{i}\right)$.

This equation can be written as a linear combination which can be learned by an Adaline neural network. $c_{1}, c_{2}, c_{3}$ and $c_{4}$ are constants representing the weights of the Adaline, as shown in figure 3.

$$
i_{d}(t) \cdot i_{i}(t)=\mathbf{W}^{T} \mathbf{X}
$$

$\mathbf{W}$ and $\mathbf{X}$ are respectively the weight vector and input vector composed of cosine and sine components of the fundamental harmonic and are given by:

$$
\mathbf{W}=\left[\begin{array}{c}
c_{1} \\
c_{2} \\
c_{3} \\
c_{4}
\end{array}\right]=\frac{I_{d} \cdot I_{i}}{2}\left[\begin{array}{c}
\cos \left(\phi_{d}-\phi_{i}\right) \\
\sin \left(\phi_{d}-\phi_{i}\right) \\
\cos \left(\phi_{d}+\phi_{i}\right) \\
\sin \left(\phi_{d}+\phi_{i}\right)
\end{array}\right] ;
$$

$$
\mathbf{X}=\left[\begin{array}{c}
\cos \left(\hat{\theta}_{d}-\hat{\theta}_{i}\right) \\
-\sin \left(\hat{\theta}_{d}-\hat{\theta}_{i}\right) \\
-\cos \left(\hat{\theta}_{d}+\hat{\theta}_{i}\right) \\
\sin \left(\hat{\theta}_{d}+\hat{\theta}_{i}\right)
\end{array}\right]
$$

The Adaline is trained with Widrow-Hoff learning algorithm and the angular phase between the two current signals is deduced from the first weight:

$$
\left(\phi_{d}-\phi_{i}\right)=\arccos \left(\frac{2}{I_{d} \cdot I_{i}} W_{1}\right)
$$

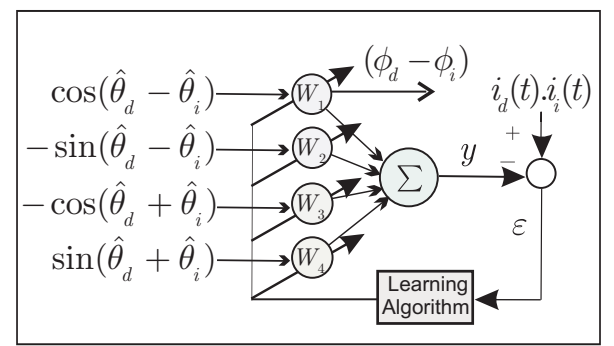

Fig. 3. Adaline for phase angle estimation between direct and inverse currents.

\section{B. Basic consideration of the faults classification}

During fault conditions, the fault classification algorithm is based on the phase angle between the direct and the inverse sequence component currents. Figure 4 shows the direct and inverse phasor diagrams of the current related to the phase $a$ to ground fault $(a-g) . i_{a d}, i_{a i}$ are the direct and inverse component fault currents of the phase $a$. Similarly, current components for phases $b$ and $c$ are denoted as $i_{b d}, i_{b i}$ and $i_{c d}$, $i_{c i}$, respectively.

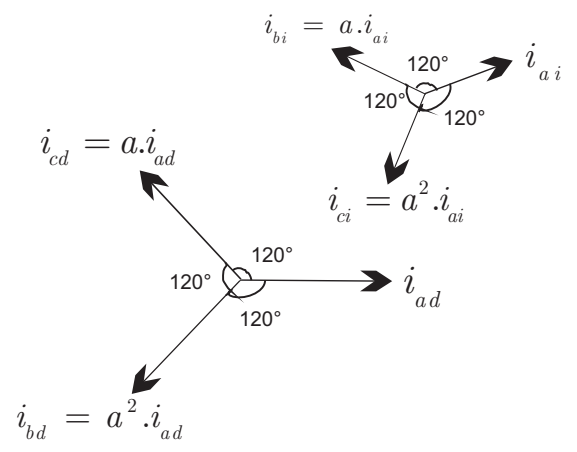

Fig. 4. Phasor diagram for $(a-g)$ fault of direct and inverse currents

Table I shows the fundamental phase angles between the direct and the inverse current components of phases $a, b$, and c for the unloaded system. $A n g_{a}=\left(\phi_{a d}-\phi_{a i}\right), A n g_{b}=$ $\left(\phi_{b d}-\phi_{b i}\right), A n g_{c}=\left(\phi_{c d}-\phi_{c i}\right)$. Nine different fault types can be observed, three single line to ground faults, three double line to ground faults and three double line faults. The symbol $\mathrm{g}$ represents the ground. The triple line to ground fault is considered as a symmetrical fault and there is no appearance of inverse and homopolar components. This fault is not included in this table. 
TABLE I

FUNDAMENTAL PHASE ANGLES RELATION FAULTS

\begin{tabular}{|l||l|l|l|}
\hline Type of fault & $\operatorname{Ang}_{a}\left({ }^{\circ}\right)$ & $\operatorname{Ang}_{b}\left({ }^{\circ}\right)$ & $\operatorname{Ang}_{c}\left({ }^{\circ}\right)$ \\
\hline \hline Phase a-g & 0 & 120 & 120 \\
\hline Phase b-g & 120 & 0 & 120 \\
\hline Phase c-g & 120 & 120 & 0 \\
\hline \hline Phases a-b & 60 & 60 & 180 \\
\hline Phases b-c & 180 & 60 & 60 \\
\hline Phases a-c & 60 & 180 & 60 \\
\hline \hline Phases a-b-g & 60 & 60 & 180 \\
\hline Phases b-c-g & 180 & 60 & 60 \\
\hline Phases a-c-g & 60 & 180 & 60 \\
\hline
\end{tabular}

\section{RESULTS AND DISCUSSION}

\section{A. Symmetrical components extraction with Adaline method}

The diagram of the system under study is shown in Figure 1. It is a sample transmission line extending between two sources. Parameters of this system are described in the Appendix.

The online symmetrical components extraction based Adaline method takes the measured three phase currents with a sampling time $T=0.01 \mathrm{~ms}$. Three kinds of faults are simulated (single phase to ground fault, two phases without ground fault, and three phases to ground fault) for the following conditions: fault time $t=0.04 \mathrm{~s}$, fault resistance $R_{f}=20 \Omega$, fault distance $L=50 \mathrm{~km}$ and load angles $\delta_{S 1}=0^{\circ}$ for first source $\mathrm{S} 1$ and $\delta_{S 2}=10^{\circ}$ for the second source $\mathrm{S} 2$.

Figure 5 shows a one phase to ground fault $(a-g)$. We notice that when the fault occurs, the symmetrical component currents are recovered. The three phase unbalanced currents are decomposed into three balanced direct, inverse and homopolar currents estimated by the Adaline neural network. The three homopolar currents are equal in magnitude and in phase. The Adaline neural network gives a stable response after a half of cycle. We can also notice that after the disappearing of the fault at $t=0.25 \mathrm{~s}$, the system returns to its normal conditions. Only the direct component of the current remains (inverse and homopolar currents are equal to zero).

Figure 6 shows a two phases without ground fault $(b-c)$. However, when the fault occurs, only the direct and inverse components current appear. The homopolar components appear instantly at the beginning and at the end of the fault (at $t=0.25 \mathrm{~s})$. It is transient and disappears after $50 \mathrm{~ms}$.

Figure 7 shows a three phases to ground fault $(a-b-c-$ $g$ ). This kind of fault is considered as a balanced fault and only the direct component current exists. The inverse and the homopolar component currents appear at the beginning of the fault and disappear at the end of the fault.

Further simulations have been carried out to test the efficiency and the robustness of our method by changing the line length and source parameters. Figure 8 shows the results for a $400 \mathrm{~km}$ line and $315 \mathrm{kV}$ voltage, the source resistance and inductance are respectively $R_{s}=0 \Omega$ and $L_{s}=46,671 \mathrm{mH}$. A single fault $(a-g)$ occurs at the middle of the line at $t=0.04$ s with $R_{f}=20 \Omega$ and the load angle $\delta_{S 2}=30^{\circ}$. Symmetrical components are recovered after $3 / 4$ of cycle. This proves the generalization ability of our method.

We notice that the response of our algorithm to faults occurring between phases takes more time than to faults
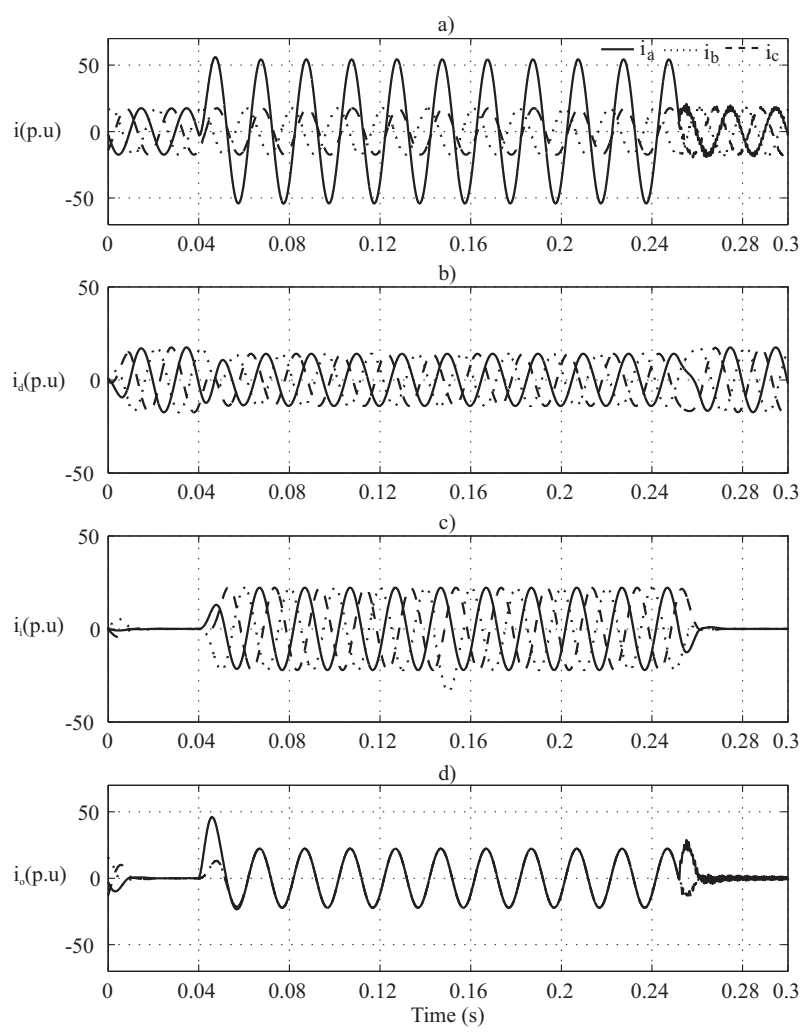

Fig. 5. One phase to ground fault $(a-g)$, located at $L=50 \mathrm{~km}$ from source S1 and at time $t=0.04 \mathrm{~s}$ with $R_{f}=20 \Omega, \delta_{S 2}=10^{\circ}$, a) three phases currents, b) direct currents, c) inverse currents, d) homopolar currents.
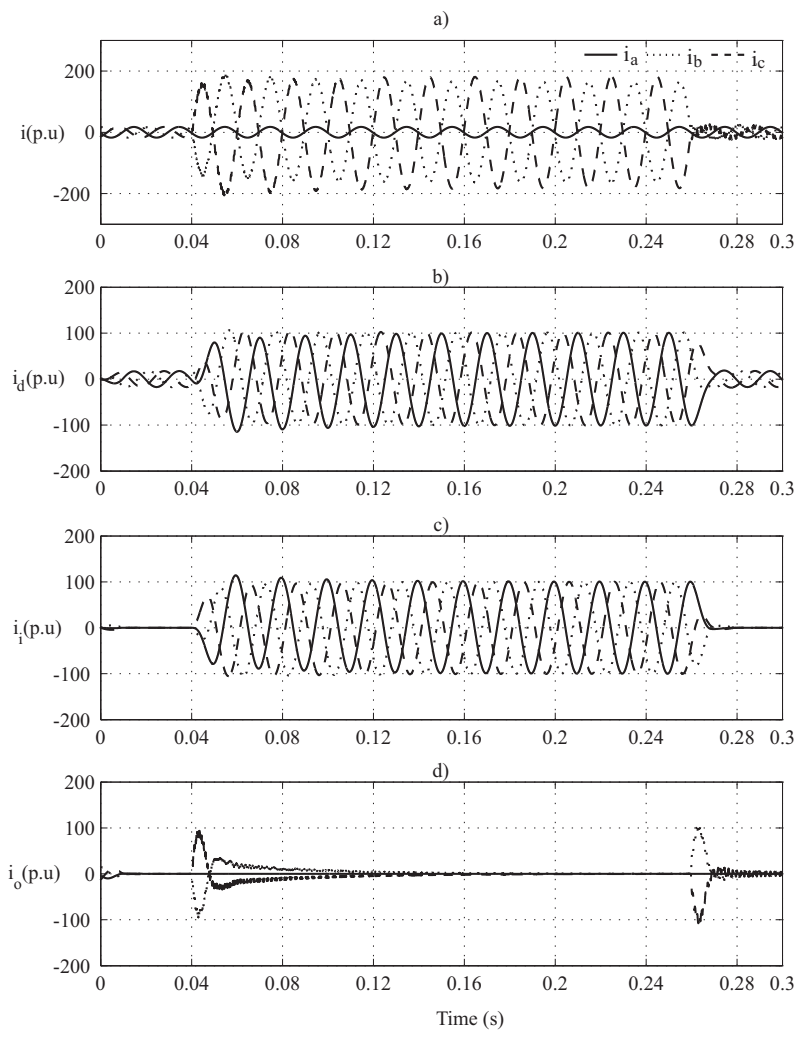

Fig. 6. Two phase fault without ground $(b-c)$, located at $L=50 \mathrm{~km}$ from source $\mathrm{S} 1$ and at time at $t=0.04 \mathrm{~s}$ with $\delta_{S 2}=10^{\circ}$, a) three phases currents, b) direct currents, c) inverse currents, d) homopolar currents. 

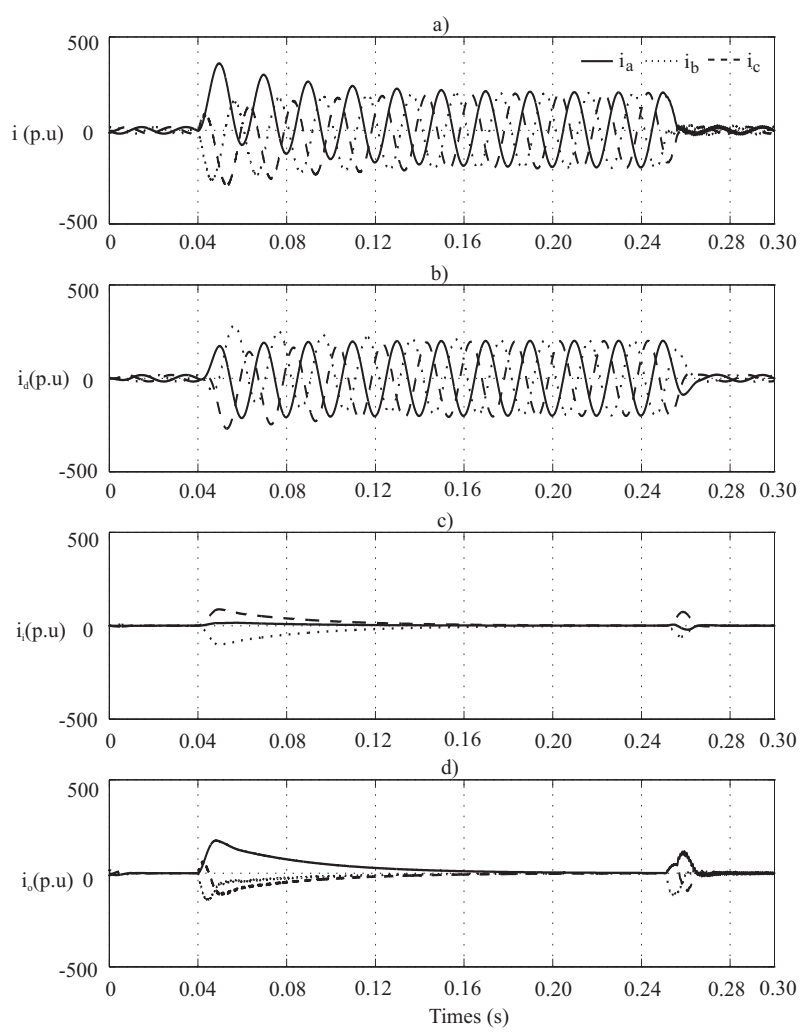

Fig. 7. Three phase fault to ground $(a-b-c-g)$, located at $L=50 \mathrm{~km}$ from source S1 and at time $t=0.04 \mathrm{~s}$ with $R_{f}=20 \Omega, \delta_{S 2}=10^{\circ}$ a) three phases currents, b) direct currents, c) inverse currents, d) homopolar currents.

TABLE II

TIME TO GET THE ESTIMATED PHASE ANGLE FOR A SINGLE PHASE TO GROUND FAULTS

\begin{tabular}{|l|l|l|l|l|l|}
\hline Type of fault & $L(\mathrm{~km})$ & $R_{f}(\Omega)$ & $\theta\left(^{\circ}\right)$ & $\delta_{S 2}\left(^{\circ}\right)$ & Time $(\mathrm{ms})$ \\
\hline \hline Phase a-g & 20 & 20 & 0 & 5 & 15 \\
\hline Phase b-g & 40 & 40 & 90 & 10 & 15 \\
\hline Phase c-g & 60 & 50 & 180 & 20 & 50 \\
\hline Phase a-g & 50 & 20 & 90 & 10 & 25 \\
\hline Phase b-g & 80 & 5 & 225 & 30 & 23 \\
\hline Phase c-g & 50 & 20 & 90 & 10 & 20 \\
\hline Phase c-g & 70 & 20 & 0 & 10 & 15 \\
\hline Phase b-g & 10 & 20 & 180 & 15 & 12 \\
\hline
\end{tabular}

occurring between phases and ground. This is due to the presence of higher harmonics and DC component in the first case. This is caused by the mutual impedance between phases.

Table II represents the results of a single phase to ground fault covering different faults: fault distance $(L)$, fault resistance $\left(R_{f}\right)$, inception angle $(\theta)$ and load angle $\left(\delta_{S 2}\right)$. The time to get the estimated Adaline components is given in the last column.

The application of Fourier algorithm for a single phase to ground fault, in our system, requires between a half to one cycle dynamic response (depending on the window length). The definition of the window length is a compromise between speed and precision. Fourier algorithm with short window (half cycle) is very fast but with minimum precision, the algorithm with long window (one cycle or more) has good precision but is slower. The dynamic response of the Adaline method takes a half cycle for this type of fault with a good compromise
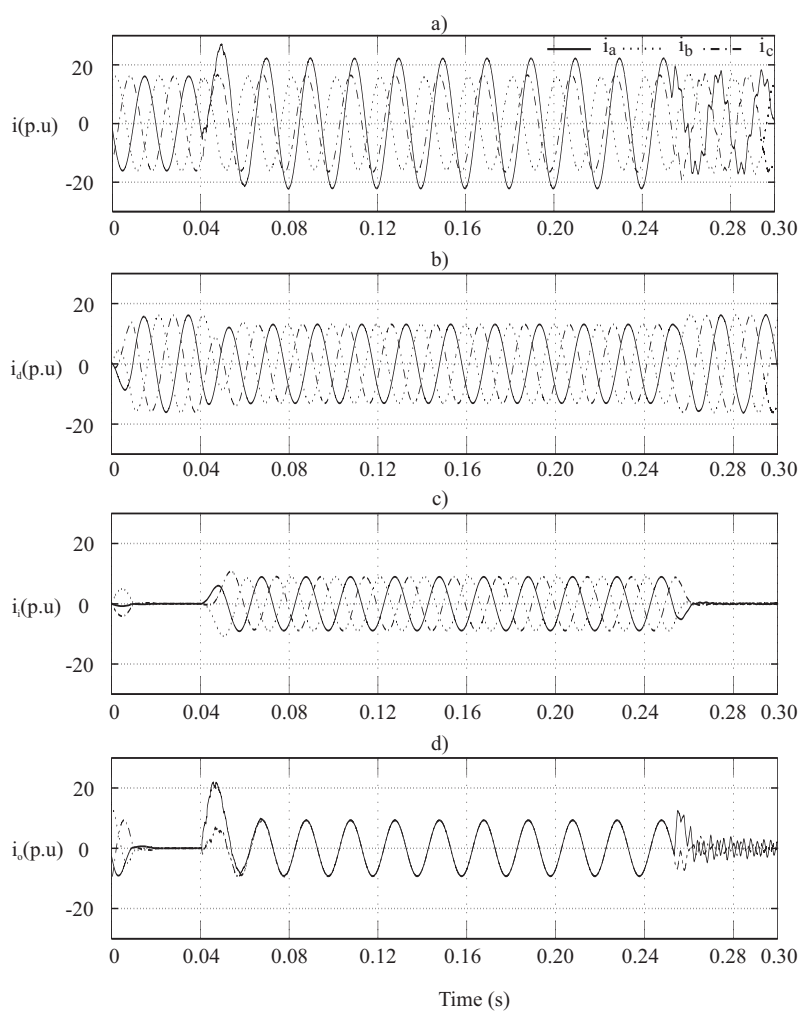

Fig. 8. One phase to ground fault $(a-g)$, located at $L=200 \mathrm{~km}$ from source S1 and at time $t=0.04 \mathrm{~s}$ with $R_{f}=20 \Omega, \delta_{S 2}=30^{\circ}$, a) three phases currents, b) direct currents, c) inverse currents, d) homopolar currents.

between speed and precision. This compromise depends on the choice of the learning rate $\alpha$ of the Adaline. In the symmetrical components identification $\alpha$ is fixed to 0.01 . This value is motivated by several tests and input types of the Adaline which are the cosine and sine (they vary between -1 to 1 ). The advantage of the Adaline is the possibility to introduce a priori knowledge of the signal in its architecture. In our case, we used the Fourier decomposition of powers in the $\mathrm{p}-\mathrm{q}$ frame. This allows to take into account the presence of harmonics, DC components and potential non-linearity of current and voltage transformers.

\section{B. Performance of the Adaline for the angular estimation}

Figure 9 shows the Adaline direct and inverse current components product $\left(i_{d}(t) \cdot i_{i}(t)\right)$ tracking and the output error $(\epsilon)$ for two phases to ground fault $(a-c-g)$ for the following fault conditions: $L=50 \mathrm{~km}, R_{f}=20 \Omega, t=0.04 \mathrm{~s}$, and $\delta_{S 2}=10^{\circ}$. The Adaline uses sine and cosine as inputs to learn the linear expression $i_{d}(t) \cdot i_{i}(t)$. Table III shows the dynamic response and the static error of the Adaline phase angle estimation for four different values of the learning rate $\alpha$. A choice of $\alpha$ is an important issue. A high value of the learning rate $\alpha$ of the Adaline accelerates convergence but may causes system instability. A small value of $\alpha$ stabilizes the system but slows down the convergence. An intermediate value is a good compromise. In our case, the compromise between the dynamic response and the static error is obtained by fixing $\alpha$ to 0.01 . 
TABLE III

ADALINE PERFORMANCES IN FUNCTION OF $\alpha$ VALUES

\begin{tabular}{|l||l|l|}
\hline value of $\alpha$ & Dynamic response & Static error \\
\hline \hline 0.005 & $34 \mathrm{~ms}$ & $2.1 \%$ \\
\hline 0.01 & $13 \mathrm{~ms}$ & $2.2 \%$ \\
\hline 0.1 & $12 \mathrm{~ms}$ & $3.5 \%$ \\
\hline 0.3 & $11 \mathrm{~ms}$ & $6.3 \%$ \\
\hline
\end{tabular}
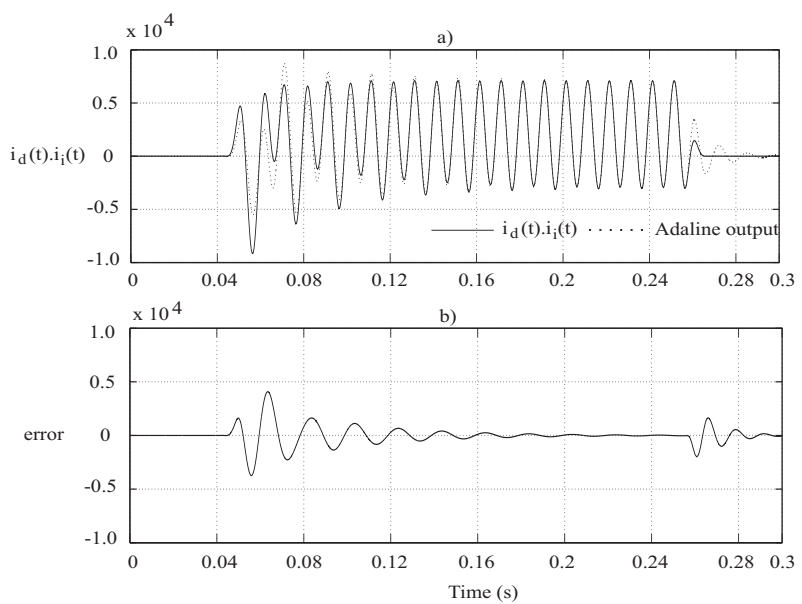

Fig. 9. a) Adaline tracking of $i_{d}(t) \cdot i_{i}(t)$ for two phases to ground fault $(a-c-g)$, b) output error.

At the appearance of the fault $(0.04 \mathrm{~s})$, the output of the Adaline tracks the product signal. After three periods, the learning error is reduced significantly. Figure 10 shows the evolution and the convergence of the Adaline weights ( $\left.W_{1}, W_{2}, W_{3}, W_{4}\right)$ and the estimated value of the RMS product $\left(I_{d} . I_{i}\right)$. The weights are stabilized after three periods. At the disappearance of the fault (at $0.25 \mathrm{~s}$ ), the neural network weights go back to zero. By replacing the first weight $W_{1}$ and the estimated $I_{d} . I_{i}$ in the equation (26), we can find the angle between the direct and inverse component currents.

The Adaline neural network is very simple and can adapt its parameters to the power supply evolution.
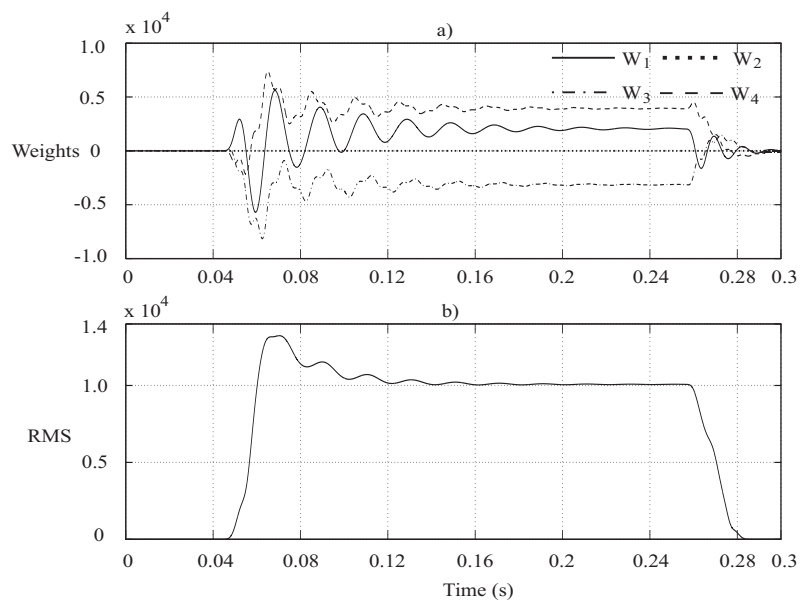

Fig. 10. a) Adaline weights for two phases to ground fault $(a-c-g)$, b) $I_{d} . I_{i}$ current RMS of phase a.
TABLE IV

Phase ANGLES ESTIMATED By AdALINE

\begin{tabular}{|l||l|l|l|}
\hline Type of fault & $\operatorname{Ang}_{a}\left({ }^{\circ}\right)$ & $\operatorname{Ang}_{b}\left({ }^{\circ}\right)$ & $A n g_{c}\left({ }^{\circ}\right)$ \\
\hline \hline Phase a-g & 1.68 & 121.7 & 118.3 \\
\hline Phase b-g & 118.3 & 1.68 & 121.7 \\
\hline Phase c-g & 121.7 & 118.3 & 1.68 \\
\hline \hline Phases a-b & 59.98 & 60.05 & 180 \\
\hline Phases b-c & 180 & 59.98 & 60.05 \\
\hline Phases a-c & 60.19 & 180 & 60.12 \\
\hline \hline Phases a-b-g & 54.11 & 65.93 & 175.3 \\
\hline Phases b-c-g & 174.5 & 54.11 & 65.91 \\
\hline Phases a-c-g & 66.07 & 180 & 54.25 \\
\hline
\end{tabular}

TABLE V

Phase angles estimated for A-B-G FAult by VARying $L, R_{f}$ And $t$

\begin{tabular}{|l|l|l||l|l|l|}
\hline$L(\mathrm{~km})$ & $R_{f}(\Omega)$ & $t(\mathrm{~s})$ & $\operatorname{Ang} g_{a}\left(^{\circ}\right)$ & $\operatorname{Ang}\left(^{\circ}\right)$ & $A n g_{c}\left({ }^{\circ}\right)$ \\
\hline \hline 5 & 20 & 0.06 & 58.61 & 61.39 & 178.7 \\
\hline 10 & 40 & 0.04 & 61.21 & 58.79 & 178.9 \\
\hline 10 & 60 & 0.125 & 62.12 & 57.9 & 176.8 \\
\hline 10 & 80 & 0.11 & 62.6 & 57.42 & 176.7 \\
\hline 35 & 20 & 0.06 & 61.72 & 58.03 & 180 \\
\hline 65 & 20 & 0.06 & 66.03 & 54.09 & 177.6 \\
\hline 95 & 20 & 0.06 & 71.74 & 48.5 & 170.8 \\
\hline
\end{tabular}

\section{Phase angles calculated by Adalines for different faults}

Table IV shows the phase angles estimated by the Adaline for all fault types in an unloaded system. Faults occur at $t=$ $0.04 \mathrm{~s}$ and at the middle of the transmission line $(L=50 \mathrm{Km})$ with a fault resistance $R_{f}=20 \Omega$. By comparing the results in Table IV with the theoretical values of Table I, we see that the phase angles estimated by the neural approach are almost similar to those calculated theoretically. Table V shows the phase angles estimated by Adaline for two phases to ground fault $(a-b-g)$ by taking the load angle $\delta_{S 2}=10^{\circ}$ and varying the fault distance $L$, fault resistance $R_{f}$ and fault time $t$. In this case, the phase angle estimation does not depend much on $R_{f}$ and $t$ variations. However, for faults farthest from the first source, the fault angle estimation is less accurate.

Thus, a complete fault classification module using the Adaline neural network can be used to classify all the different fault types in $\mathrm{HV}$ transmission lines, by considering different fault locations, fault resistance and different fault times.

\section{CONCLUSION}

This paper presents a new approach to extract symmetrical components currents and phase angles in HV transmission lines based on Adaline neural networks. The basic idea is to implement the Adaline as an online adaptive fault classifier module. The symmetrical current components extraction is based on a new formulation issued of the Instantaneous Power Theory (IPT) which consists of the powers decomposition in the $\alpha \beta$ frame. Four Adalines are used to separate the average and oscillating active and reactive terms of the powers. The direct and inverse sequence currents are recovered by converting the resulting average terms in the three phase frame. By using these symmetrical components, three other Adalines are proposed to learn the linear expression of the direct and inverse sequence current products. Thus, the phase angles can be determined and used to classify the different fault types in 
HV transmission lines. The Matlab SimPower System Toolbox is used to generate and test the proposed algorithms in different cases. Simulation results shows that the two Adaline neural network approaches presented in this paper provide a good identification of the symmetrical components currents and the phase angles. This method is able to adapt to online varying parameters and is suitable for protective relay improvement.

APPENDIX

SYSTEM PARAMETERS

\begin{tabular}{|l||l|}
\hline Sources voltage $(V)$ & $200 \mathrm{Kv}$ \\
\hline Frequency $(f)$ & $50 \mathrm{~Hz}$ \\
\hline Sources resistance $\left(R_{s}\right)$ & $0.8929 \Omega$ \\
\hline Sources inductance $\left(L_{s}\right)$ & $16.58 \mathrm{mH}$ \\
\hline Line length $(L)$ & $100 \mathrm{~km}$ \\
\hline Direct-sequence impedance $\left(Z_{d}\right)$ & $12.73+j 293 \mathrm{~m} \Omega / \mathrm{km}$ \\
\hline Homopolar-sequence impedance $\left(Z_{o}\right)$ & $386.4+j 1295.7 \mathrm{~m} \Omega / \mathrm{km}$ \\
\hline Direct-sequence capacitance $\left(C_{d}\right)$ & $12.74 \mathrm{nF} / \mathrm{km}$ \\
\hline Homopolar-sequence capacitance $\left(C_{o}\right)$ & $7.751 \mathrm{nF} / \mathrm{km}$ \\
\hline
\end{tabular}

\section{REFERENCES}

[1] A.G. Phadke, and J.S. Thorp, Computer Relaying for Power Systems, 2nd ed., Wiley Ltd, England, 2009.

[2] F.H.J. Altuve, V.I. Diaz, and M.E Vazquez, Fourier and Walsh digital filtering algorithms for distance protection, IEEE Transactions on Power Systems, vol. 11 , n. 1, pp. 457-462, Feb. 1996.

[3] J.A. Jiang, C.s. Chen, and C.W. Liu, A New Protection Scheme for Fault Detection, Direction Discrimination, Classification, and Location in Transmission Lines, IEEE Transactions Power Delivery, vol. 18, no. 1, pp. 34-42, Jan. 2003.

[4] M. Enns, L. Budler, T.W. Cease, A. Elneweihi, E. Guro, M. Kezunovic, J. Linders, P. Leblanc, J. Postforoosh, R. Ramaswami, F. Soudi, R. Taylor, H. Ungrad, S.S. Venkata, and J. Zipp, Potential applications of expert systems to power system protection, IEEE Transactions on Power Delivery, vol. 9 , no. 2, pp. 720-728, 1994.

[5] K. Kawahara, H. Sasaki, J. Kubokawa, M. Kitagawa, and H. Sugihara, Expert system for designing transmission line protection system, International Journal of Electrical Power and Energy Systems, vol. 17, no. 1, pp. 69-78, Feb. 1995.

[6] A. Ferrero, S. Sangiovanni, and E. Zappitelli, A fuzzy-set approach to fault type identification in digital relaying, IEEE Transactions Power Delivery, vol. 10, no. 1, pp. 169-175, Jan. 1995.

[7] B. Das, and J.V. Reddy, Fuzzy logic based fault classification scheme for digital distance protection IEEE Transactions Power Delivery, vol. 20, no. 2, pp. 609-616, Apr. 2005.

[8] R.N. Mahanty, and P.B. Dutta Gupta, A fuzzy logic based fault classification approach using current samples only Electric Power Systems Research, vol. 77, no. 5-6, pp. 501-507, Apr. 2007.

[9] O.A.S. Youcef, Combined fuzzy logic wavelet based Fault Classification technique for Power System relaying, IEEE Transactions Power Delivery, vol. 19, no. 2, pp. 582-589, Apr. 2004.

[10] S.P. Valsan, and K.S. Swarup, Wavelet transform based digital protection for transmission lines, International Journal of Electrical Power and Energy Systems, vol. 31, no. 7-8, pp. 379-388, Sep. 2009.

[11] P.K. Dash, D.P. Swain, A. Routray, A.C. Liew, An adaptive neural network approach for the estimation of power system frequency, Electric Power Systems Research, vol. 41, no. 3, pp. 203-210, Jun. 1997.

[12] D. Ould Abdeslam, P. Wira, J. Merckl’e, D. Flieller and Y.-A. Chapuis, $A$ Unified Artificial Neural Network Architecture for Active Power Filters, IEEE Transactions on Industrial Electronics, vol. 54, no. 1, pp. 61-76, Feb. 2007.

[13] D. Flieller, D. Ould Abdeslam, P. Wira, and J. Mercklé, Distortions identification and compensation based on artificial neural networks using symmetrical components of the voltages and the currents, Electric Power Systems Research, vol. 79, no. 7, pp. 1145-1154, Jul. 2009.

[14] H. Akagi, E.H. Watanabe, and M. Aredes, Instantaneous Power Theory and Applications to Power Conditioning, Wiley-IEEE Press, 2007.
[15] N.K. Nguyen, D. Flieller, P. Wira, D. Ould Abdeslam, Neural Network for phase and symmetrical components estimation in power systems, 35th annual Conference of the IEEE Industrial Electronics Society (IECON09), Porto, Portugal, pp. 3-5, Nov. 2009.

[16] B. K. Bose, Neural Network Applications in Power Electronics and Motor Drives-An Introduction and Perspective, IEEE Transactions on Industrial Electronics, vol. 54, no. 1, pp. 14-33, Feb. 2007.

[17] T. Dalstein, B. Kulicke, Neural Network Approach to Fault Classification for High Speed Protective Relaying, IEEE Transactions on Power Delivery, vol. 10, no. 2, pp.1002-1009, Apr. 1995.

[18] T. Bouthiba, Fault detection and classification technique in EHV transmission lines based on artificial neural networks, European Transactions on Electrical Power Journal, vol. 15, no. 5, pp 443-454, 2005.

[19] M.S. Pasand, H.K. Zadeh, and O.P. Malik, Transmission line fault detection and phase selection using ANN, International Conference on Power Systems transients - IPST, New Orleans, USA, 2003.

[20] E.A. Mohamed, H.A. Talaat, and E.A. Khamis, Fault diagnosis system for tapped power transmission lines, Electric Power Systems Research, vol. 80, no. 5, pp. 599-613, May. 2010.

[21] R.K. Aggarwal, Q.Y. Xuan, R.W. Dunn, A.T. Johns, and A. Bennet, A novel fault classification technique for double-circuit lines based on a combined unsupervised/supervised neural network, IEEE Transactions Power Delivery, vol. 14, no. 4, pp. 1250-1256, Oct. 1999.

[22] W.M. Lin, C.D. Yang, J.H. Lin, and M.T. Tsay, A fault classification method by RBF neural network with OLS learning procedure, IEEE Transactions Power Delivery, vol. 16, no. 4, pp. 473-477, Oct. 2001.

[23] Y. H. Song, Q. X. Xuan, and A. T. Johns, Comparison studies of five neural network based fault classifiers for complex transmission lines, Electric Power Systems Research, vol. 43, no. 2, pp. 125-132, Nov. 1997.

[24] P.K. Dash, A.K. Pradhan, and G. Panda, Application of minimal radial basis function neural network to distance protection, IEEE Transactions Power Delivery, vol. 16, no. 1, pp. 68-74, Jan. 2001.

[25] H. Wang and W.W.L. Keerthipala, Fuzzy-neuro approach to fault classification for transmission line protection, IEEE Transactions Power Delivery, vol. 13, no. 4, pp. 1093-1104, Oct. 1998.

[26] S. Vasilic and M. Kezunovic, Fuzzy ART neural network algorithm for classifying the power system faults, IEEE Transactions Power Delivery, vol. 20, no. 2, pp. 1306-1314, Apr. 2005.

[27] S.M. Yeo, C.H. Kim, K.S. Hong, Y.B. Lim, R.K. Aggarwal, A.T. Johns, and M.S. Choi, A novel algorithm for fault classification in transmission lines using a combined adaptive network and fuzzy inference system, Electrical Power and Energy Systems, vol. 25, pp. 747-758, 2003.

[28] J. Sadeh, and H. Afradi, A new and accurate fault location algorithm for combined transmission lines using Adaptive Network-Based Fuzzy Inference System, Electric Power Systems Research, vol. 79, no. 11, pp. 1538-1545, Nov. 2009

[29] M.J. Reddy, and D.K. Mohanta, A DSP based frequency domain approach for Classification of Transmission Line Faults, Digital Signal Processing, vol. 18, no. 5, pp. 751-761, Sep. 2008.

[30] P.K. Dash, and S.R. Samantaray, A novel distance protection scheme using time frequency analysis and pattern recognition approach, International Journal of Electrical Power and Energy Systems, vol. 29, no. 2, pp. 129-137, Feb. 2007.

[31] F.L. Yousfi, D. Ould Abdeslam, N.K. Nguyen, Adaline for fault detection in Electrical High Voltage Transmission Line, 36th annual Conference of the IEEE Industrial Electronics Society (IECON10), Glendale, AZ, USA, pp.1963-1968, 2010.

[32] O. Karacasu, and M.H. Hocaoglu, An Adaline based arcing fault detection algorithm for single-pole autoreclosers, Electric Power Systems Research, vol. 81, no. 2, pp. 367-376, Feb. 2011.

[33] B. Widrow, and M.A. Lehr, 30 years of adaptive neural networks: perceptron, Madaline, and back propagation, Proceedings of the IEEE, vol. 79, no 9, pp. 1415-1442, Sep. 1990.

Fatima Louisa Yousfi received the M.Sc. degree in electrical engineering from the University of Science and Technologie of Oran (USTO), Algeria, in 2002. She has been a lecturer at USTO since 1995. She is now working towards the Ph.D. degree at the University of Haute-Alsace, Mulhouse, France. Her research interest is the power system protection by using artificial neural networks. 
Djaffar Ould Abdeslam (M'08) received the engineer degree in electronic engineering from the University of Tizi-Ouzou, Algeria, in 2000, and the M.Sc. degree in electrical engineering from the University of Franche-Comté, Besançon, France, in 2002, and the Ph.D. degree from the University of HauteAlsace, Mulhouse, France, in 2005. He is currently an Associate Professor in the University of Haute-Alsace. His research interest includes artificial neural networks applied to power active filters and power electronics.

Tahar Bouthiba is a Professor of electrical engineering. He was born in 1959, and has been a lecturer at the University of Science and Technology of Oran (USTO) since 1987. His research interests include computer relaying and control switching using digital techniques and artificial intelligence.

Ngac Ky NGUYEN received his B.Sc degree in electrical engineering from the Ho Chi Minh City University of Technology (HCMUT), Viet Nam in 2005 and his M.Sc. from Poly'Tech Nantes, University of Nantes, France in 2007 and his Ph.D. from the University of Haute-Alsace (UHA), France in 2010, both in electrical and electronic engineering. He works actually at the electrical engineering department, INSA of Strasbourg, France. His research interests are the control of active power filters and synchronous motors with artificial neural networks.

Jean Mercklé received the M.Sc. and Ph.D. degrees in electrical engineering from University Nancy I, Nancy, France, in 1982 and 1988, respectively. In 1988, he joined the MIPS Laboratory, University of Haute-Alsace, Mulhouse, France, where he participated in several adaptive signal processing projects. From 1991 to 1993, he was with the Department of Electrical and Computer Engineering, University of California at San Diego, contributing to a 3$\mathrm{D}$ optoelectronic neural architecture with efficient learning. He is currently a Professor of Electrical and Computer Engineering. His research interests include adaptive neural computation with application to power electronic systems control. 